\title{
Pediatric Pes Planus: A Review
}

\author{
Maria Elena Cucuzza ${ }^{1}$, Angela D'ambra' ${ }^{1}$, GiuseppeEvola ${ }^{2}$ and Francesco Roberto Evola ${ }^{2}$ \\ ${ }^{1}$ University of Catania, Pediatric Clinic, Via Plebiscito 626, Catania, Italy \\ ${ }^{2}$ University Of Catania, Via Plebiscito 626, Catania, Italy
}

Received: September 15, 2017; Published: September 21, 2017

*Corresponding author: Maria Elena Cucuzza, University of Catania, Via Plebiscito 628, Catania, Italy, Email : me.cucuzza@gmail.com

\begin{abstract}
Flat foot is a complex three-dimensional skeletal disorder with multifactorial etiology frequently encountered in evolutionary age, and tends to resolve spontaneously in adolescence. Despite the high frequency, there is no precise and universally accepted flat foot definition due to the absence of clinical and radiographic diagnostic criteria [1,2]. From the anatomical point of view it is characterized by: hyperpronation and valgus of the hindfoot (due to the eversion of the subtalar joint); abduction and supination of the forefoot and reduction of the plantar vault. Often the flat foot is associated with brevity of the achilles tendon. Risk factors are joint laxity, male sex, severe knee-rotation, and obesity [3]. All children have a flat foot at birth because there is a fat pad at the base of the foot in order to protect the skeletal structures. Normally, flat foot resolves at the age of 10 , following the development of the longitudinal medial plantar vault. In some cases it is also found in adulthood. Morley found a flat foot incidence of $97 \%$ at 2 years, and $4 \%$ after 10 years [4]; Staheli, through a study of 800 patients, reports the same incidence and states that it dramatically reduces between 3 and 6 years [5]. Evans and Harris state that the incidence of flat foot in the adult is respectively $15 \%$ and $20 \%$ [6].
\end{abstract}

Keywords: Pes Planus; Children; Management; Review

\section{Biomechanical and Classification}

The foot is a complex structure of the body responsible of the body support during the dynamic and static phase of the movement. The foot is composed by two components: the "talar" portion, consisting of talus, navicular bone, cuneiforms and I-IIII metatarsals, has a dynamic function during the walk, and a "calcaneus" consisting of calcaneum, cuboid, IV -V metatarsals, responsible of the load in the static phase of walking. In addition, the hindfoot acts as a rigid lever arm of the propulsion phase of the movement. The plantar vault consists of three arches: medial longitudinal arch, lateral longitudinal arch and transversal arch; the lowering of the plantar vault is often caused by a reduction in the medial longitudinal arch. The development and the function of the foot are strictly regulated by sensory and mechanical receptors located in the subtalar joint.

Flat foot can be classified as flexible or rigid; the flexible foot is the most common form (95\%) and it is secondary to capsuleligament laxity, decreased muscle strength, obesity, Spring ligament insufficiency or altered limb alignment; the rigid foot (5\%) is caused by a congenital vertical talus, tarsal coalescence (astragalocalcaneal and calcaneo-navicular), trauma, juvenile arthritis, neuro-muscular dysfunction. Some authors define the flexible flat foot as "physiological" when it is asymptomatic and without functional alterations [1]. In the flexible foot the plantar vault is there without load and disappears under load, while in the rigid foot the reduction of the plantar vault is not affected by the load. Rigid flat foot can be accompanied by pain, due to a reduction of the subtalar articulation. This is often an expression of congenital bone deformities (accessory navicular), accompanied by neuromuscular disorders (cerebral palsy and myelomeningocele) [7].

\section{Clinical Examination}

Suspect of flat foot is often reported by parents due to abnormal wear and tear of the shoes of their kids and rapid fatigue after sport or prolonged standing. Clinical examination starts with the evaluation of the lower limb alignment, with particular attention to the rotation of the hips (often anteversion) and knees (often in internal rotation), the presence of generalized joint laxity (Marfan Syndrome or connective tissue disorders), the brevity of the achilles tendon (Silfverskiold test), the shape of the foot with and without load, and the presence of pain. Foot pain in this case may be either medial, localized in the midfoot or plantar fascia, or lateral, at the sinus tarsi; in flat feet with short achilles tendon the pain is caused by the tendon shortening.

Clinical examination should be performed by observing the front, back and lateral profiles of the lower limbs. In the front profile there is a medial load of the foot with abduction of the forefoot, internal rotation of the tibia and convergent patellar strabism, valgus deformity of the hallux (in the most severe forms); in the 
posterior profile there is a pronation of the hindfoot, the forefoot protruds laterally (sign of too many toes); in the medial profile there is a reduction of the plantar vault and a double projection caused by the internal rotation of the talus (double malleolus) and the medial projection of the navicular bone (third malleolus).

The clinical examination is accompanied by functional evaluation tests of the reducibility of the flat-footedness and the examination of the plantar imprint on the podoscope. The most used tests of reducibility are the "jack test" or "bending test", which is the forced flexion of the toe, and the "toe raising test", which consists of raising the foot in the tips; in both tests the elevation of the medial longitudinal arch of the plantar vault and the supination of the hindfoot allow to differentiate a rigid foot from a flexible.

When examining the footprint with podoscope, is observed enlargement of isthmus, the intermediate portion that separates the forefoot from hindfoot.

\section{Diagnostic Exams}

For diagnosis it is necessary to perform the X-ray examination in orthostatic position, which allows to measure the inclination angles of the bones. The most widely used angles are the Kite angle $\left(<25^{\circ}\right)$ delimited between the longitudinal axis of the talus and that of the heel; the angle of Costa-Bertani $\left(<125^{\circ}\right)$, delimited between the two lines formed by the talus-navicular joint and directed towards the calcanear tuberosity and the sesamoid bone of the first metatarsus; the angle of Mary $\left(<20^{\circ}\right)$, formed by the longitudinal axis of the talus with the first metatarsus in lateral view of $x$-ray.

Foot TC examination is done in the presence of rigid flat feet to evaluate the presence of bone or fibrous sinostosis between the tarsal bones and to organize the respective surgical treatment [2].

\section{Treatment}

Treatment of rigid flat foot is exclusively surgical and is based on the removal of the congenital bone deformity. The use of orthoses in the rigid foot may worsen the symptoms of the patient [8].

For flexible flat foot, therapy varies depending on whether the foot is asymptomatic or symptomatic. The asymptomatic foot does not require any treatment; there are no studies that show that any (surgical and non-surgical) treatment can affect normal foot development [8]. Studies in literature are not prospective, without any control group, they have small and non-homogeneous samples, with heterogeneous diagnostic criteria, with variable follow-up, which lids to discordant results [2,9]. Asymptomatic flexible flat foot is often treated by mistake with costs that are not justified by literature studies [6].
The use of orthosis in the asymptomatic foot in 3 to 8 years of age has not been shown to improve foot development, and in some cases has caused psychological dependencies [10]. Asymptomatic flexible foot surgery is indicated for patients at risk of developing articular pain (anterior knee pain, low back pain) and arthrosis of the foot in adulthood, although the literature does not indicate precisely which patients are at risk of such complication.

Initial treatment of flat asymptomatic foot is non-surgical and it is based on rest, reduction in sports activity, medical therapy and rehabilitation. Although in literature orthotics does not seem to alter the foot development, studies say that it can achieve symptomatic pain relief [3]. Surgical treatment is indicated in case of failure of the conservative treatment and is based on: soft tissue reconstruction (medial foot capsular-tightening, tendon transfers, peroneus brevis or achilles tendon lengthening), realignment osteotomies (calcaneal osteotomy, calcaneus-medial cuneiformcuboid osteotomy) and arthroereisis [1].

Soft-tissue procedures have shown poor results and are often associated with other surgical procedures; osteotomies, though technically complex and with satisfactory clinical results, may lead to early arthrosis of the foot.

Arthroereisis, which is particularly widespread among orthopedics since it is rapid and minimally invasive, it is based on a surgical limitation of subtalar articolarity by inserting metallic or synthetic implants in the sinus tarsi. This procedure allows to eliminate the pain, preventing the posterior tibial tendon dysfunction and improving the shape of the foot; the main complications are talar neck fracture, subtalar fusion, malpositioning or extrusion of implant, under correction or overcorrection, foreign body-reaction, inflammatory reaction, and persistent foot pain.

\section{Conclusion}

Most flexible flat feet are asymptomatic, do not cause pain or disability in adulthood, and require no treatment. Surgical treatment is indicated only for relapsing pain after failure of the conservative treatment with orthoses.

There are no extensive prospective studies in literature that evaluate the results of various treatments to identify the most appropriate treatment for the flat pediatric foot. Furthermore, there are no studies that clearly identify patients who are at risk of functional pain and disability when not adequately treated surgically. 


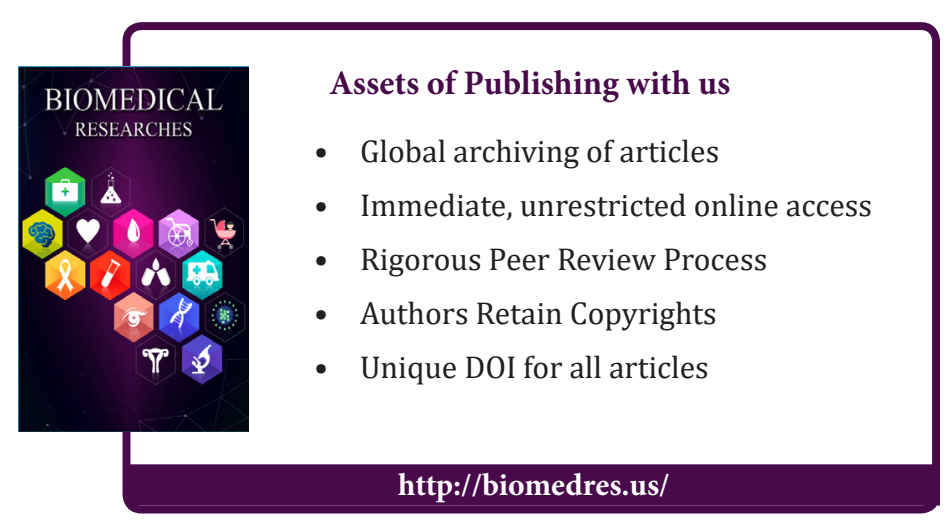

